\title{
Phosphocitrate Is Potentially a Disease-Modifying Drug for Noncrystal-Associated Osteoarthritis
}

\author{
Yubo Sun, ${ }^{1}$ David R. Mauerhan, ${ }^{1}$ Atiya M. Franklin, ${ }^{1}$ James Norton, \\ Edward N. Hanley Jr., ${ }^{1}$ and Helen E. Gruber ${ }^{1}$ \\ ${ }^{1}$ Department of Orthopedic Surgery, Carolinas Medical Center, P.O. Box 32861, Charlotte, NC 28232, USA \\ ${ }^{2}$ Department of Biostatistics, Carolinas Medical Center, P.O. Box 32861, Charlotte, NC 28232, USA
}

Correspondence should be addressed to Yubo Sun; yubo.sun@carolinas.org

Received 18 December 2012; Accepted 10 January 2013

Academic Editor: Andre Van Wijnen

Copyright (C) 2013 Yubo Sun et al. This is an open access article distributed under the Creative Commons Attribution License, which permits unrestricted use, distribution, and reproduction in any medium, provided the original work is properly cited.

Phosphocitrate (PC), a calcification inhibitor, inhibits the development of crystal-associated osteoarthritis (OA) in Hartley guinea pigs. However, the molecular mechanisms underlying its disease-modifying effect remain elusive. This study sought to test the hypothesis that PC has calcium crystal-independent biological activities which are, at least in part, responsible for its diseasemodifying activity. We found that PC inhibited the proliferation of OA fibroblast-like synoviocytes in the absence of calcium crystals. Consistent with its effect on cell proliferation, PC downregulated the expression of numerous genes classified in cell proliferation. PC also downregulated the expression of many genes classified in angiogenesis and inflammatory response including prostaglandin-endoperoxide synthase 2, interleukin-1 receptor, type I, and chemokine (C-C motif) ligand 2. In contrast, PC upregulated the expression of many genes classified in musculoskeletal tissue development, including aggrecan, type I collagen, and insulin-like growth factor binding protein 5 . These findings suggest that PC is not only a promising disease-modifying drug for crystal-associated OA but also for noncrystal-associated OA.

\section{Introduction}

Osteoarthritis (OA) is a progressive disorder characterized by the degeneration of articular cartilage, formation of osteophytes, and synovial lining hyperplasia. It is one of the most prevalent causes of disability in the aging population and has enormous economic and social consequences. However, existing treatment options only provide symptomatic relief and have no effect on the progression of the underlying disease. The lack of progress in the development of diseasemodifying drugs for OA therapy is largely due to our limited understanding of the pathogenesis of the disease and our insufficient knowledge about the molecular targets for intervention.

The most apparent pathological changes in OA are usually found in articular cartilage. Synovium of patients with OA has traditionally been considered to be normal and is used as control in studies to investigate the pathological changes in the synovium of patients with rheumatoid arthritis (RA)
$[1,2]$. OA fibroblast-like synoviocytes (FLSs) have also been used as control cells in some studies $[3,4]$. However, synovial lining hyperplasia and inflammation, a potential leading cause of knee pain, are common findings in OA patients $[5,6]$. Studies have demonstrated that OA synovium and OA FLSs display different gene expression profiles compared with the synovium and FLSs derived from normal control subjects $[7,8]$. These findings indicate that OA synovium plays an important role in the pathogenesis of OA.

Basic calcium phosphate (BCP) crystal and calcium pyrophosphate dihydrate (CPPD) crystal are the two most common intra-articular crystals $[9,10]$. The presence of these crystals in the synovial fluid or joint tissues of end-stage OA patients is a well-recognized event [11-14]. In cell culture, calcium crystals induced mitogenesis, production of matrix metalloproteinases (MMPs), and endocytotic activity of cells [15-18]. However, the clinical significance of these calcium crystals and their role in the development and/or progression of OA remain unclear $[14,19-21]$. 
Phosphocitrate (PC), a potent anticalcification molecule, was originally identified in mammalian mitochondria [22]. PC inhibits the formation and growth of calcium crystals by its strong binding to amorphous calcium phosphates aggregates and the surface of calcium crystals. In cell cultures, PC inhibited crystal-induced mitogenesis, expression of MMPs, and cell death [23-25]. In Hartley guinea pig model of crystal-associated OA, PC inhibited meniscal calcification and reduced the degeneration of articular cartilage [26]. These findings appear to provide support for the notions that calcium crystals play an important role in the development and/or progression of $\mathrm{OA}$ and that calcification inhibitors are promising disease-modifying drugs for crystal-associated OA therapy [27]. However, a bisphosphonate, a potent calcification inhibitor similar to PC, failed to inhibit the development of $\mathrm{OA}$ in Hartley guinea pigs, raising questions about the role of crystals in the development of crystal-associated OA [28]. Several studies indicate that PC has biological activities unrelated to its anticalcification activity. For example, PC reduced the degradation of low-density lipoprotein by $60 \%$ [29]. PC inhibited transforming growth factor- $\beta 1$-induced proliferation of progressive ankylosis fibroblasts [30]. We decide to perform this study to test the hypothesis that PC has unique crystal-independent biological activities which are responsible, at least in part, for its disease-modifying activity.

\section{Materials and Methods}

Dulbecco's modified eagle medium (DMEM), fetal bovine serum (FBS), and stock antibiotic/antimycotic mixture were products of Invitrogen (Carlsbad, CA, USA). PC was synthesized according to the procedure previously described [31].

2.1. Cell Cultures. Telomerase-transduced human OA FLSs, or hTERT-OA 13A FLSs, have been previously described [8]. Primary OA FLSs were prepared from synovial tissues collected with the approval of the authors' institutional review board from OA patients undergoing total joint replacement surgery at our medical center. The need for informed consent was waived because the synovial tissues were surgical waste, and no private patient information was collected. Briefly, synovial tissues were minced into small pieces $(3 \mathrm{~mm} \times$ $3 \mathrm{~mm}$ ) and cultured in $100 \mathrm{~mm}$ plates at $37^{\circ} \mathrm{C}$ in DMEM containing $10 \%$ FBS. Every three days, culture medium was changed. When OA FLSs reached $80 \%$ confluence, they were harvested and passaged. Human foreskin fibroblasts were obtained from ATCC (CRL-2429, Mananas, USA) and expanded in DMEM containing 10\% FBS.

2.1.1. Cell Proliferation. hTERT-OA 13A FLSs $\left(4 \times 10^{4}\right)$ were plated in six-well cluster plates. On the second day, DMEM containing $10 \%$ FBS and PC was added to the top three wells. DMEM containing 10\% FBS without PC was added to bottom three wells. The culture medium was changed every three days until the cells in the bottom wells without PC reached $85 \%$ confluence. All cells were then harvested and cell numbers were determined by cell count using a hemocytometer.
This proliferation assay was also performed using primary OA FLSs and foreskin fibroblasts for comparison.

2.2. RNA Extraction. hTERT-OA $13 \mathrm{~A}$ FLSs were plated in four $100 \mathrm{~mm}$ plates at $90 \%$ confluence. On the second day, DMEM containing $1 \%$ FBS was added. On the third day, DMEM in two plates was replaced with DMEM containing $1 \%$ FBS and PC at a final concentration of $0.6 \mathrm{mM}$. DMEM in other two plates was replaced with DMEM containing $1 \%$ FBS but without PC. Twenty-four hours later, total RNA was extracted from these cells using Trizol reagent (Invitrogen, Carlsbad, CA, USA) and purified using Oligotex kit (Qiagen, Valencia, CA, USA). These RNA samples were used for microarray analyses and RT-PCR experiments.

2.3. Microarray. Double-stranded DNA was synthesized using SuperScript double-stranded cDNA synthesis kit (Invitrogen, Carlsbad, CA, USA). The DNA product was purified using GeneChip sample cleanup module (Affymetrix, Santa Clara, CA, USA). cRNA was synthesized and biotin labeled using BioArray high yield RNA transcript labeling kit (Enzo Life Sciences, Farmingdale, NY, USA). The cRNA product was purified using GeneChip sample cleanup module and subsequently chemically fragmented. The fragmented, biotinylated cRNA was hybridized to HG-U133_Plus_2 gene chip using Affymetrix Fluidics Station 400 (Affymetrix, Santa Clara, CA, USA). The fluorescent signals were quantified during two scans by Agilent Gene Array Scanner G2500A (Agilent Technologies, Palo Alto, CA, USA) and GeneChip operating Software (Affymetrix, Santa Clara, CA, USA). Genesifter software (VizX Labs, Seattle, WA, USA) was used for the analysis of differential gene expression and gene ontology.

2.4. Real-Time RT-PCR. Briefly, cDNA was synthesized using TaqMan Reverse Transcription Reagents (Applied Biosystems, Inc., University Park, IL, USA) using the RNA samples described. Quantification of relative transcript levels of selected genes was performed using ABI7000 Real-Time PCR system (Applied Biosystems, Inc., University Park, IL, USA). TaqMan Gene Expression assays (Applied Biosystems, Inc., University Park, IL, USA) were used. cDNA samples were amplified with an initial Taq DNA polymerase activation step at $95^{\circ} \mathrm{C}$ for 10 minutes, followed by 40 cycles of denaturation at $95^{\circ} \mathrm{C}$ for 15 seconds and annealing at $60^{\circ} \mathrm{C}$ for one minute. Fold change was calculated, and the expression level of the genes was normalized to the expression level of the housekeeping gene glyceraldehyde 3-phosphate dehydrogenase (GAPDH) according to the method described [32]. The experiments were performed independently using RNA samples extracted from both hTERT-OA 13A FLSs and primary OA FLSs.

2.5. Statistical Analysis. Data are expressed as the mean $\pm \mathrm{SD}$. For cell proliferation, the difference between two experimental groups was analyzed using Student's $t$-test. For real-time RT-PCR, experiment was repeated twice in triplicates. The difference between two experimental groups 
TABLE 1: Differentially expressed genes in PC-treated hTERT-OA 13A FLS compared with the untreated cells.

\begin{tabular}{|c|c|c|c|c|}
\hline Biological process & $\begin{array}{l}\text { Gene } \\
\text { name }\end{array}$ & Gene ID & $\begin{array}{l}\text { Differ. } \\
\text { expre. } \\
(\text { fold })^{*}\end{array}$ & Description \\
\hline \multirow{31}{*}{ Cell proliferation } & BLM & NM_000057 & -3.64 & Bloom syndrome \\
\hline & CCNE2 & AF112857 & -3.74 & Cyclin E2 \\
\hline & CCNE1 & AI671049 & -2.30 & Cyclin E1 \\
\hline & CDC25A & AY137580 & -3.63 & Cell division cycle 25 homolog A (S. pombe) \\
\hline & CDC25C & NM_001790 & -2.31 & Cell division cycle 25 homolog C (S. pombe) \\
\hline & $\mathrm{CDC} 2$ & AA749427 & -3.13 & Cell division cycle $2, \mathrm{G} 1$ to $\mathrm{S}$ and $\mathrm{G} 2$ to $\mathrm{M}$ \\
\hline & CDC6 & NM_001254 & -2.36 & Cell division cycle 6 homolog (S. cerevisiae) \\
\hline & $\mathrm{CDC} 7$ & NM_003503 & -2.12 & Cell division cycle 7 homolog (S. cerevisiae) \\
\hline & CDCA5 & BE614410 & -2.41 & Cell division cycle associated 5 \\
\hline & CDCA8 & ВC001651 & -2.11 & Cell division cycle associated 8 \\
\hline & $\mathrm{CDK} 2$ & AB012305 & -2.74 & Cyclin-dependent kinase 2 \\
\hline & NCAPH & D38553 & -2.64 & Non-SMC condensin I complex, subunit H \\
\hline & HELLS & NM_018063 & -2.49 & Helicase, lymphoid specific \\
\hline & AURKB & AB011446 & -2.43 & Aurora kinase B \\
\hline & KIF23 & AW192521 & -2.41 & Kinesin family member 23 \\
\hline & CLASP2 & ВC029035 & -2.40 & Cytoplasmic linker-associated protein 2 \\
\hline & NUF2 & AF326731 & -2.35 & NUF2, NDC80 kinetochore complex component, homolog \\
\hline & DSN1 & NM_024918 & -2.35 & DSN1, MIND kinetochore complex component, homolog \\
\hline & SPC24 & AI469788 & -2.32 & SPC24, NDC80 kinetochore complex component, homolog \\
\hline & SPC25 & AF225416 & -2.10 & SPC25, NDC80 kinetochore complex component, homolog \\
\hline & HMGA2 & AI990940 & -2.30 & High mobility group AT-hook 2 \\
\hline & LIG1 & NM_000234 & -2.25 & Ligase I, DNA, ATP-dependent \\
\hline & KIFC1 & BC000712 & -2.21 & Kinesin family member $\mathrm{Cl}$ \\
\hline & BRCA2 & X95152 & -2.18 & Breast cancer 2, early onset \\
\hline & ERCC6L & NM_017669 & -2.17 & Excision repair cross-complement repair deficiency, comp. group 6 like \\
\hline & SPAG5 & NM_006461 & -2.16 & Sperm-associated antigen 5 \\
\hline & NEK2 & $\mathrm{Z} 25425$ & -2.14 & Never-in-mitosis-gene-A- (NIMA) related kinase 2 \\
\hline & NCAPG & NM_022346 & -2.12 & Non-SMC condensin I complex, subunit G \\
\hline & ZWINT & NM_007057 & -2.01 & ZW10 interactor antisense \\
\hline & PARD3B & AF428251 & 3.24 & Par-3 partitioning defective 3 homolog B (C. elegans) \\
\hline & 11-Sep & AI333326 & 2.28 & Septin 11 \\
\hline \multirow{11}{*}{ Angiogenesis } & NRP1 & AF280547 & -2.69 & Neuropilin 1 \\
\hline & TEK & BF594294 & -2.58 & TEK tyrosine kinase, endothelial \\
\hline & ELK3 & NM_005230 & -2.42 & ELK3, ETS-domain protein (SRF accessory protein 2) \\
\hline & EREG & NM_001432 & -1.90 & Epiregulin \\
\hline & PML & AW291023 & -1.89 & Promyelocytic leukemia \\
\hline & COL15A1 & NM_001855 & -1.80 & Collagen, type XV, alpha-1 \\
\hline & NRP2 & AI819729 & -1.75 & Neuropilin 2 \\
\hline & SPHK1 & NM_021972 & -1.72 & Sphingosine kinase 1 \\
\hline & FOXC2 & NM_005251 & -1.68 & Forkhead box C2, mesenchyme forkhead 1 (MFH-1) \\
\hline & SCG2 & NM_003469 & -1.66 & Secretogranin II (chromogranin C) \\
\hline & EDNRA & NM_001957 & -1.56 & Endothelin receptor type A \\
\hline
\end{tabular}


TABle 1: Continued.

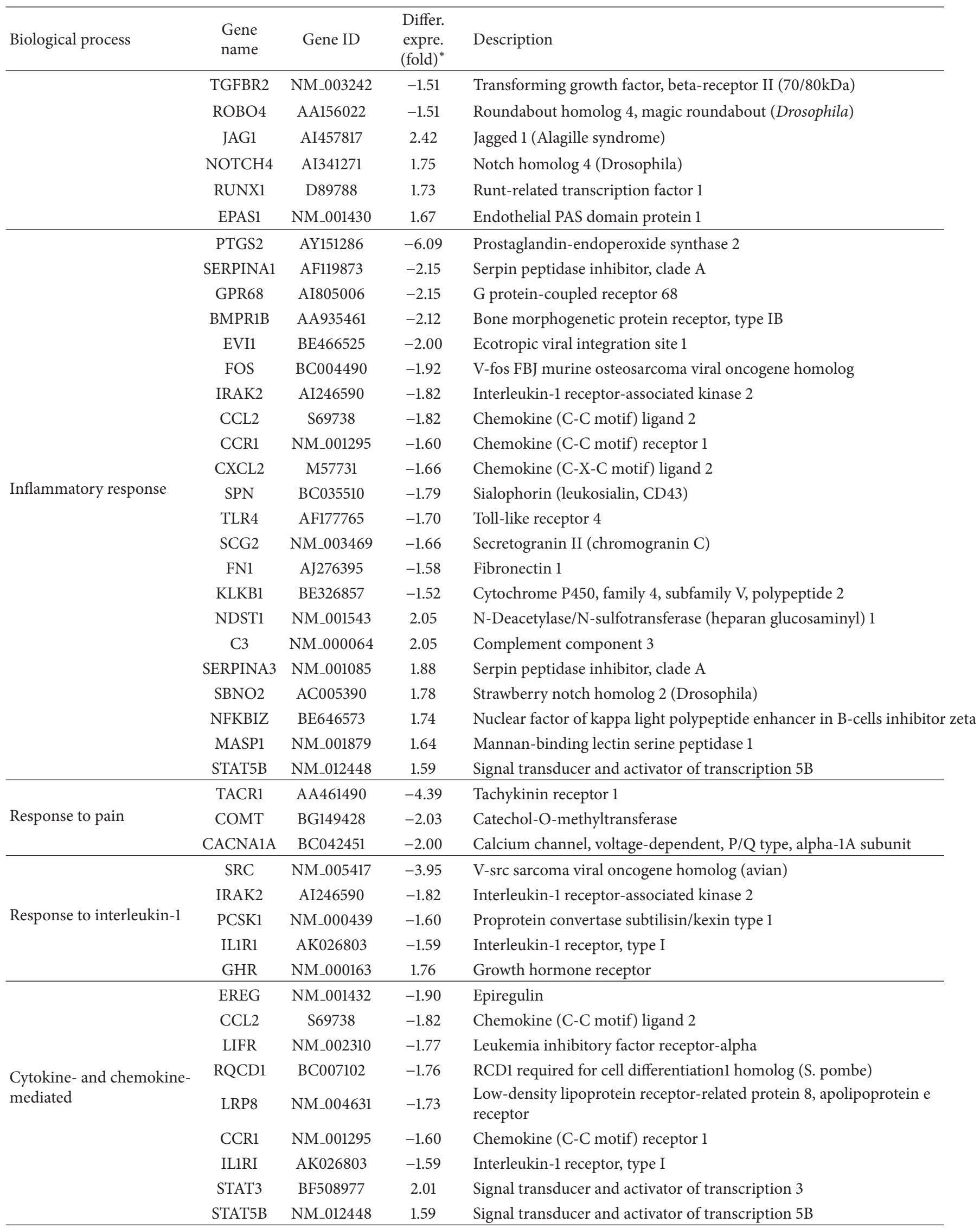


TABle 1: Continued.

\begin{tabular}{lcccl}
\hline Biological process & $\begin{array}{c}\text { Gene } \\
\text { name }\end{array}$ & Gene ID & $\begin{array}{l}\text { Differ. } \\
\text { expre. } \\
(\text { fold })^{*}\end{array}$ & Description \\
\hline & BCL10 & AA994334 & -2.38 & B-cell CLL/lymphoma 10 \\
& TNFAIP3 & AI738896 & -2.07 & Tumor necrosis factor, alpha-induced protein 3 \\
& FOXP3 & NM_014009 & -1.92 & Forkhead box P3 \\
Interleukin-6 production & EREG & NM_001432 & -1.90 & Epiregulin \\
& TOLR4 & AF177765 & -1.70 & Toll-like receptor 4 \\
& ADORA2B & NM_000676 & -1.59 & Adenosine A2b receptor \\
& IL6R & NM_000565 & 2.03 & Interleukin-6 receptor \\
\hline PLAU & K03226 & -2.21 & Plasminogen activator, urokinase \\
& PLAUR & U08839 & -1.92 & Plasminogen activator, urokinase receptor \\
PLA2G4A & M68874 & -1.51 & Phospholipase A2, group IVA (cytosolic, calcium dependent) \\
PTGER4 & AA897516 & -1.91 & Prostaglandin E receptor 4 (subtype EP4) \\
& EGR1 & AI459194 & -2.25 & Early growth response 1 \\
& FOS & BC004490 & -1.92 & V-fos FBJ murine osteosarcoma viral oncogene homolog \\
& FOSL1 & BG251266 & -1.95 & FOS-like antigen 1 \\
THBS1 & BF084105 & 2.41 & Thrombospondin 1 \\
\hline
\end{tabular}

${ }^{*}$ Negative number indicates decreased expression (fold) in PC-treated hTERT-OA 13A FLS compared with the untreated cells. Positive number indicates elevated expression (fold) in PC-treated hTERT-OA 13A FLS compared with the untreated cells.

was analyzed using Student's $t$-test. In all cases, $P$ values less than 0.01 were considered significant. Statistical analysis was performed using the SAS software, version 9.3.

\section{Results}

3.1. PC Inhibited Proliferation of OA FLSs. PC had no effect on the proliferation of human foreskin fibroblasts (Figure 1(a), the left bar group). However, PC inhibited the proliferation of hTERT-OA 13A FLSs (Figure 1(a), the right bar group). There were about $60 \%$ fewer hTERT-OA 13A FLSs in the PC-treated wells after nine days of culture compared with the cells in the untreated wells $(P<0.01)$.

Next, we examined the effect of PC on cell proliferation using primary OA FLSs. For comparison, we also examined the effect of disodium salt of ethane-1-hydroxy-1, 1bisphosphonic acid (EHDP), which is a bisphosphonate. As shown in Figure 1(b), both PC and EHDP inhibited the proliferation of primary OA FLSs in a dose-dependent manner. The morphologies of PC-treated OA FLSs and untreated OA FLSs were similar (not shown), indicating that the reduction in cell number was not due to cellular toxicity of PC.

3.2. Effect of PC on Gene Expression. Microarray analysis revealed that of the more than 50,000 transcripts examined, 3,011 transcripts displayed significant differential expression (more than 1.5-fold) between the PC-treated hTERT-OA 13A FLSs and the untreated hTERT-OA 13A FLSs; 1,558 transcripts were downregulated, and 1,453 transcripts were upregulated by PC. Differentially expressed genes were classified according to gene ontology category biological process. The genes that fell into specific biological processes previously implicated in $\mathrm{OA}$, or suspected to have a role in $\mathrm{OA}$ are listed in Tables 1 and 2.

As shown in Table 1 , the expression of numerous genes classified in cell proliferation was downregulated by PC. Of the 32 differentially expressed genes with more than twofold changes between the PC-treated cells and untreated cells, the expression of 30 genes, including cyclin E1 (CCNE1; -2.30fold change), cyclin E2 (CCNE2; -3.74-fold change), and cell division cycle 25 homolog $\mathrm{C}$ (CDC25C; -2.31-fold change), was downregulated by PC. This downregulatory effect of PC on the genes associated with cell proliferation is consistent with the finding that PC inhibited the proliferation of OA FLSs (Figure 1).

The expression of many genes classified in angiogenesis and inflammatory response was also downregulated by PC. Of the 18 differentially expressed genes classified in angiogenesis, the expression of 13 genes, including neuropilin 1 (NRP1; -2.69-fold change) and TEK tyrosine kinase (TEK; -2.58 -fold change), was downregulated by PC. Of the 22 differentially expressed genes classified in inflammatory response, the expression of 15 genes, including prostaglandin-endoperoxide synthase $2 /$ cyclooxygenase (PTGS2/Cox-2; -6.09-fold change), chemokine (C-C motif) ligand 2 (CCL2/MCP-1; -1.82-fold change), and chemokine (C-X-C) ligand 2 (CXCL2; 1.66-fold change), was downregulated by PC. In addition, of the 3 differentially expressed genes classified in response to pain, the expression of all 3 genes, including tachykinin receptor 1 (TACR1, -4.39), was downregulated by PC. Of the 5 differentially expressed genes classified in the response to interleukin-1 (IL-1), the expression of 4 genes, including $\mathrm{V}$-src sarcoma viral oncogene homolog (SRC, -3.95-fold change), IL-1 receptor type 1 (IL1R1; -1.59fold change), and IL-1 receptor-associated kinase 2 (IRAK2; -1.82 -fold change), was downregulated by PC. 


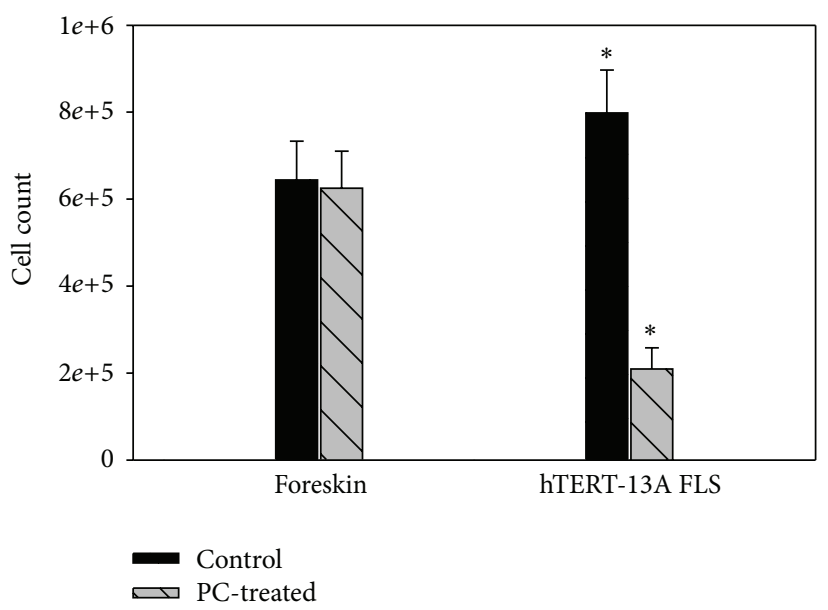

(a)

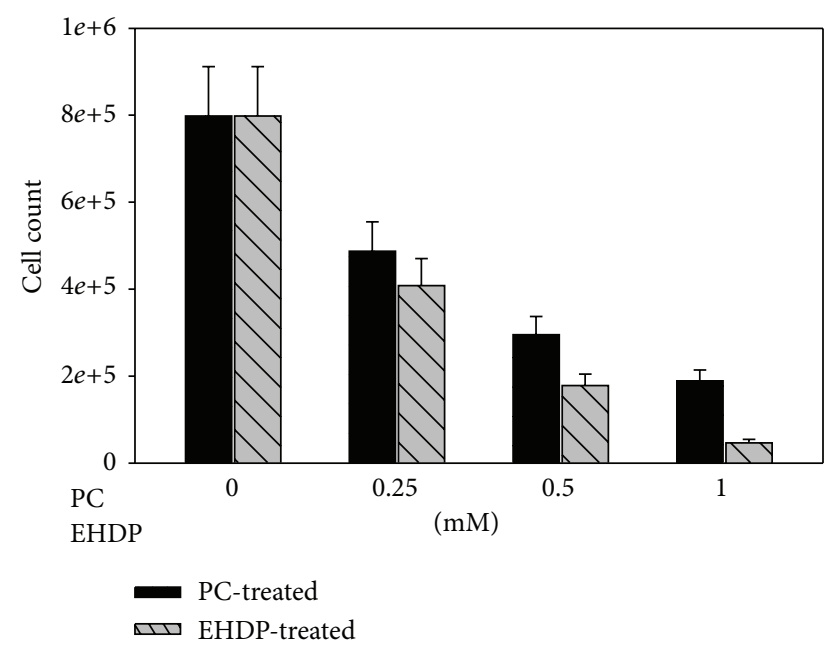

(b)

FIGURE 1: PC inhibits the proliferation of OA FLSs. (a)There were about $65 \%$ fewer hTERT-OA 13A FLSs in the PC-treated wells after nine days of culture compared to those in the untreated wells $(P<0.01)$. (b) PC and EHDP inhibited the proliferation of primary OA FLSs in a dose-dependent manner.

Furthermore, the expression of many genes classified in the cytokine- and chemokine-mediated signal pathway and IL-6 production was downregulated by PC (Table 1). Of the 9 differentially expressed genes classified in the cytokine and chemokine-mediated signal pathway, the expression of 7 genes, including chemokine (C-C motif) receptor 1 (CCR1, -1.60 -fold change), was downregulated by PC. Of the 7 differentially expressed genes classified in IL-6 production, the expression of 6 genes, including tumor necrosis factor, alphainduced protein 3 (TNFAIP3, -2.07-fold), was downregulated by PC.

Most of the genes upregulated by PC fell into biological processes associated with musculoskeletal tissue development. For example, of the 25 differentially expressed genes classified in muscle tissue development, the expression of 15 genes, including insulin-like growth factor binding protein 5 (IGFBP5; 8.57-fold change), was upregulated by PC. Of the 28 differentially expressed genes classified in skeletal development, the expression of 18 genes, including annexin A2 (2.17-fold change), vitamin D (1,25- dihydroxyvitamin D3) receptor (VDR; 2.11-fold change), aggrecan (ACAN; 1.80 fold change), collagen type 1 , alpha-1 (COL1A1; 1.66-fold change), and collagen type XI alpha-1 (COL11A1; 1.50-fold change), was upregulated by PC (Table 2).

3.3. Real-Time RT-PCR. The genes selected for validation for the differential expression between the PC-treated and untreated hTERT-OA 13A FLSs by real-time RT-PCR were listed in Table 3. As shown, the differential expressions of the genes examined were confirmed by real-time RT-PCR.

\section{Discussion}

Previous studies demonstrated that PC inhibited BCP crystalstimulated mitogenesis and expression of PTGS2/Cox-2 [15,
33, 34]. These observations provided the bases for the hypothesis that PC is potentially a disease-modifying drug for crystal-associated OA. However, the findings presented in this study clearly indicate that the inhibitory activities of PC on mitogenesis and proliferation of OA FLSs and the downregulatory activity of $\mathrm{PC}$ on the expression of PTGS2/Cox-2 are intrinsic properties of PC. These distinct biological activities of PC are not dependent on the presence of calcium crystals.

Synovial hyperplasia is associated with the severity of knee pain and fast hyaline articular cartilage loss in OA $[35,36]$. Synovial hyperplasia is also associated with synovial angiogenesis [37]. OA FLSs, but not normal control FLSs, induced a significant enhancement of angiogenesis [38]. These observations indicate that OA FLSs play an important role in the pathogenesis of OA. In this study, we demonstrated that PC not only downregulated the expression of numerous genes associated with cell proliferation but also downregulated the expression of many genes associated with angiogenesis. These findings indicate that $\mathrm{PC}$ is potentially an antisynovial hyperplasia agent.

PTGS2/Cox-2 is a molecular target for the management of arthritis pain and inflammation [39, 40]. The strong downregulation of PTGS2/Cox-2 by PC suggests that PC may have some effect on the pain associated with OA. This potential effect of PC on pain is further supported by the finding that PC significantly downregulated the expression of TACR1 (-4.39-fold change) and SRC ( -3.95 -fold change) (Table 1). TACR1 is a $\mathrm{G}$ protein-coupled receptor which is upregulated in the joint tissues of patients with painful OA [41]. SRC, a tyrosine kinase, plays a role in substance $\mathrm{P}$ signaling which has been implicated in many inflammatory diseases $[42,43]$. These findings together indicate that PC is potentially an analgesic agent.

In this study, we demonstrated that PC strongly downregulated the expression of many genes classified in cytokine- 
TABLE 2: Differentially expressed genes in PC-treated hTERT-OA 13A FLS compared to the untreated cells.

\begin{tabular}{|c|c|c|c|c|}
\hline Biological process & Gene name & Gene ID & $\begin{array}{l}\text { Differ. } \\
\text { Expre. } \\
\text { (fold) }\end{array}$ & Description \\
\hline \multirow{25}{*}{ Muscle tissue development } & IGFBP5 & AW157548 & 8.57 & Insulin-like growth factor binding protein 5 \\
\hline & CACNB4 & NM_000726 & 2.73 & Calcium channel, voltage-dependent, beta- 4 -subunit \\
\hline & TPM1 & AI521618 & 2.43 & Tropomyosin 1 (alpha) \\
\hline & JAG1 & U61276 & 2.02 & Jagged 1 (Alagille syndrome) \\
\hline & MORF4L2 & H43976 & 1.90 & Mortality factor 4 like 2 \\
\hline & NRG1 & NM_013957 & 1.88 & Neuregulin 1 \\
\hline & SIRT2 & BG722779 & 1.86 & Sirtuin (silent mating type information regulation 2 homolog) 2 \\
\hline & NF1 & D12625 & 1.80 & Neurofibromin 1 \\
\hline & OBSL1 & BF446688 & 1.78 & Obscurin-like 1 \\
\hline & MBNL1 & AA732240 & 1.73 & Muscleblind-like (Drosophila) \\
\hline & TPM1 & NM_000366 & 1.72 & Tropomyosin 1 (alpha) \\
\hline & CAV2 & AA150110 & 1.67 & Caveolin 2 \\
\hline & RXRA & BE675800 & 1.66 & Retinoid X receptor, alpha \\
\hline & $\mathrm{NR} 2 \mathrm{~F} 2$ & AL554245 & 1.63 & Nuclear receptor subfamily 2, group F, member 2 \\
\hline & TCF7L2 & AV721430 & 1.61 & Transcription factor 7-like 2 (T-cell specific, HMG-box) \\
\hline & TBX2 & U28049 & -4.17 & T-box 2 \\
\hline & ADRB2 & NM_000024 & -2.36 & Adrenergic, beta-2-receptor, surface \\
\hline & SORT1 & BE742268 & -1.93 & Sortilin 1 \\
\hline & GJC1 & NM_005497 & -1.77 & Gap junction protein, gamma 1, $45 \mathrm{kDa}$ \\
\hline & CENPF & U30872 & -1.77 & Centromere protein F, 350/400 ka (mitosin) \\
\hline & BCL2 & NM_000657 & -1.71 & B-cell CLL/lymphoma 2 \\
\hline & TBX3 & U69556 & -1.71 & T-box 3 \\
\hline & $\mathrm{SDC1}$ & NM_002997 & -1.65 & Syndecan 1 \\
\hline & TBX5 & AW269421 & -1.54 & T-box 5 \\
\hline & RARB & NM_015854 & -1.51 & Retinoic acid receptor, beta \\
\hline \multirow{18}{*}{ Skeletal development } & ANXA2 & D28364 & 2.17 & Annexin A2 \\
\hline & VDR & AA772285 & 2.11 & Vitamin D (1,25-dihydroxyvitamin D3) receptor \\
\hline & GNAS & AI693143 & 1.95 & GNAS complex locus \\
\hline & ACAN & NM_001135 & 1.80 & Aggrecan \\
\hline & COL1A1 & AI743621 & 1.66 & Collagen, type I, alpha-1 \\
\hline & COL1A2 & AA628535 & 1.88 & Collagen, type I, alpha-2 \\
\hline & COL11A1 & NM_001854 & 1.50 & Collagen, type XI, alpha-1 \\
\hline & COL12A1 & AU146651 & 1.93 & Collagen, type XII, alpha-1 \\
\hline & MSX2 & D89377 & 1.85 & Msh homeobox 2 \\
\hline & GHR & NM_000163 & 1.76 & Growth hormone receptor \\
\hline & MEF2C & AL536517 & 1.59 & Myocyte enhancer factor $2 \mathrm{C}$ \\
\hline & THRA & NM_003250 & 1.57 & Thyroid hormone receptor, alpha \\
\hline & RUNX2 & AW469546 & 1.55 & Runt-related transcription factor 2 \\
\hline & CLEC3B & NM_003278 & 1.55 & Exosome component 7 \\
\hline & MEF2C & N22468 & 1.55 & Myocyte enhancer factor $2 \mathrm{C}$ \\
\hline & IGFBP4 & NM_001552 & 1.54 & Insulin-like growth factor binding protein 4 \\
\hline & PRKRA & AA279462 & 1.53 & Protein kinase, interferon-inducible RNA-dependent activator \\
\hline & TNFRSF11B & NM_002546 & 1.50 & Tumor necrosis factor receptor superfamily, member $11 \mathrm{~b}$ \\
\hline
\end{tabular}


TABLE 2: Continued.

\begin{tabular}{|c|c|c|c|c|}
\hline Biological process & Gene name & Gene ID & $\begin{array}{l}\text { Differ. } \\
\text { Expre. } \\
\text { (fold) }^{*}\end{array}$ & Description \\
\hline & BMPR1B & AA935461 & -2.12 & Bone morphogenetic protein receptor, type IB \\
\hline & ANKH & AF274753 & -1.93 & Ankylosis, progressive homolog (mouse) \\
\hline & ACVR2A & NM_001616 & -1.89 & Activin A receptor, type IIA \\
\hline & CYTL1 & NM_018659 & -1.83 & Cytokine-like 1 \\
\hline & TBX3 & U69556 & -1.71 & T-box 3 (ulnar mammary syndrome) \\
\hline & SOX9 & NM_000346 & -1.71 & SRY- (sex-determining-region-Y-) box 9 \\
\hline & FOXC2 & NM_005251 & -1.68 & Forkhead box C2 (MFH-1, mesenchyme forkhead 1) \\
\hline & KIAA1217 & BC017424 & -1.66 & KIAA1217 \\
\hline & MMP9 & NM_004994 & -1.61 & Matrix metallopeptidase 9 \\
\hline & TGFBR2 & NM_003242 & -1.51 & Transforming growth factor, beta-receptor II $(70 / 80 \mathrm{kDa})$ \\
\hline \multirow{5}{*}{$\begin{array}{l}\text { Collagen biosynthetic } \\
\text { process }\end{array}$} & COL3A1 & AU146808 & 1.83 & Collagen, type III, alpha-1 \\
\hline & COL1A2 & AA 628535 & 1.88 & Collagen, type I, alpha-2 \\
\hline & COL1A1 & AI743621 & 1.66 & Collagen, type I, alpha-1 \\
\hline & TRAM2 & AI986461 & 1.58 & Translocation-associated membrane protein 2 \\
\hline & ITGA2 & N95414 & -1.74 & Integrin, alpha-2 (CD49B, alpha-2 subunit of VLA-2 receptor) \\
\hline \multirow{5}{*}{ Collagen catabolic process } & MMP3 & NM_002422 & 3.18 & Matrix metallopeptidase 3 (stromelysin 1, progelatinase) \\
\hline & ADAMTS5 & BI254089 & 3.32 & ADAM metallopeptidase with thrombospondin type 1 motif, 5 \\
\hline & ADAMTS14 & W60649 & 2.15 & ADAM metallopeptidase with thrombospondin type 1 motif, 14 \\
\hline & MMP16 & U79292 & 1.80 & Matrix metallopeptidase 16 (membrane inserted) \\
\hline & MMP9 & NM_004994 & -1.61 & Matrix metallopeptidase 9 \\
\hline
\end{tabular}

*Negative number indicates decreased expression (fold) in PC-treated hTERT-OA 13A FLS compared with the untreated cells. Positive number indicates elevated expression (fold) in PC-treated hTERT-OA 13A FLS compared with the untreated cells.

TABLE 3: Differential expression confirmed by real-time RT-PCR.

\begin{tabular}{lcccr}
\hline Gene name & Gene ID & Differential expression microarray & Differential expression RT-PCR & Differential expression RT-PCR** $^{*}$ \\
\hline BLM & NM_000057 & -3.64 & -4.23 & -3.95 \\
HELLS & NM_018063 & -2.49 & -2.87 & -2.12 \\
CCNE2 & AF112857 & -3.74 & -3.93 & -3.70 \\
CDC25C & NM_001790 & -2.31 & -2.01 & -1.94 \\
EGR1 & AI459194 & -2.25 & -2.45 & -3.29 \\
FOSL1 & BG251266 & -1.95 & -2.31 & -2.11 \\
PLAUR & U08839 & -1.92 & -2.41 & -3.22 \\
PLA2G4A & M68874 & -1.51 & -1.86 & -2.11 \\
PTGS2 & AY151286 & -6.09 & -5.77 & -5.10 \\
TACR1 & AA461490 & -4.39 & -3.92 & -3.45 \\
CCL2 & S69738 & -1.82 & -2.02 & -1.79 \\
GPR68 & AI805006 & -2.15 & -2.54 & -2.28 \\
CACNA1A & BC042451 & -2.00 & -2.10 & -2.22 \\
IGFBP5 & AW157548 & 8.57 & 6.32 & 5.46 \\
ADAMTS5 & BI254089 & 3.32 & 2.81 & 2.11 \\
COL1A2 & AA628535 & 1.88 & 1.40 & 1.64 \\
\hline
\end{tabular}

${ }^{*}$ Differential expression-the numbers are the ratio of the relative expression level of a specific gene in PC-treated hTERT-OA 13A FLSs compared with the relative expression level of the specific gene in the untreated hTERT-OA 13A FLSs. ${ }^{* *}$ Differential expression - the numbers are the ratio of the relative expression level of a specific gene in PC-treated primary FLSs compared with the relative expression level of the specific gene in the untreated primary OA FLSs. 
and chemokine-mediated signal pathway, including IL1R1, CCL2, and CCR1. IL-1 $\beta$ is a main inflammatory cytokine found within OA joints and represents one of the possible treatment targets. CCL2 is a small chemokine which recruits leukocytes to synovial inflammation site and is central to the development of pain and inflammation associated with OA [44]. The findings presented in this study indicate that PC may inhibit inflammatory cell activation and infiltration and that $\mathrm{PC}$ is potentially an anti-inflammator agent.

We also demonstrated that PC downregulated the expression of plasminogen activator, urokinase (PLAU, -2.21-fold change), PLAU receptor (PLAUR, -1.92-fold change), early growth response 1 (EGR-1, -2.25-fold change), v-fos FBJ murine osteosarcoma viral oncogene homolog (FOS, -1.65fold change), and FOS-like antigen 1 (FOSL1, -1.87-fold change). PLAU is a serine protease which catalyzes the conversion of inactive zymogen plasminogen to active protease plasmin when binding to its receptor PLAUR. Plasmin is capable of degrading all the components of the extracellular matrix and activating other enzymes such as MMPs. Higher levels of PLAU and PLAUR were detected in arthritic specimens [45-47]. EGR-1, FOS, and FOSL1 are involved in FLSs activation and implicated in the pathogenesis of RA [4850]. The downregulation of these genes by PC supports the hypothesis that PC is potentially a disease-modifying drug for OA therapy.

Interestingly, PC upregulated the expression of many genes classified in muscle and skeletal tissue development, including IGFBP5. Previous studies demonstrated that inhibition of IGFBP5 proteolysis improved the joint architecture and reduced articular cartilage loss in a dog model of OA [51-53]. The strong upregulation of IGFBP5 by PC is clearly consistent with the notion that $\mathrm{PC}$ is potentially a diseasemodifying drug for OA therapy. Moreover, PC upregulated the expression of ACAN (1.80-fold change), COL1A1 (1.66fold change), COL1A2 (1.88-fold change), COL3A1 (1.83-fold change), COL11A1 (1.50-fold change), and COL12A2 (1.93fold change), indicating that PC may improve the integrity of synovial tissues.

Surprisingly, PC upregulated the expression of MMP3 (3.18-fold change) and ADAM metallopeptidase with thrombospondin type 1 motif 5 (ADAMTS5; 3.32-fold change) (Table 2). The upregulation of both extracellular matrix proteins and extracellular matrix protein-degrading enzymes by PC indicates that PC may promote synovial tissue repair and regeneration. The implication of this specific effect on the disease process of OA is unclear at present.

\section{Conclusions}

PC is potentially an antisynovial hyperplasia, analgesic and anti-inflammator agent. PC is not only a promising diseasemodifying drug for crystal-associated OA but also a promising disease-modifying drug for noncrystal-associated OA. The findings presented in this study provide further support for the development of PC, and/or its analogues, as diseasemodifying drugs for OA therapy.

\section{Acknowledgments}

This study is supported in part by an NC Biotech Center Grant and a Charlotte-Mecklenburg Education and Research Foundation Grant (to Y. Sun). This study was performed at Carolinas Medical Center, Charlotte, NC, USA. The authors declare no conflict of interests.

\section{References}

[1] U. Müller-Ladner, J. Kriegsmann, J. Tschopp, R. E. Gay, and S. Gay, "Demonstration of granzyme A and perforin messenger RNA in the synovium of patients with rheumatoid arthritis," Arthritis \& Rheumatism, vol. 38, no. 4, pp. 477-484, 1995.

[2] Y. T. Konttinen, T. F. Li, J. Mandelin et al., "Increased expression of extracellular matrix metalloproteinase inducer in rheumatoid synovium," Arthritis \& Rheumatism, vol. 43, pp. 275-280, 2000.

[3] W. K. Aicher, A. H. Heer, A. Trabandt et al., "Overexpression of zinc-finger transcription factor Z-225/Egr-1 in synoviocytes from rheumatoid arthritis patients," Journal of Immunology, vol. 152, no. 12, pp. 5940-5948, 1994.

[4] N. Cagnard, F. Letourneur, A. Essabbani et al., "Interleukin32, CCL2, PF4F1 and GFD10 are the only cytokine/chemokine genes differentially expressed by in vitro cultured rheumatoid and osteoarthritis fibroblast-like synoviocytes," European Cytokine Network, vol. 16, no. 4, pp. 289-292, 2005.

[5] M. D. Smith, S. Triantafillou, A. Parker, P. P. Youssef, and M. Coleman, "Synovial membrane inflammation and cytokine production in patients with early osteoarthritis," The Journal of Rheumatology, vol. 24, no. 2, pp. 365-371, 1997.

[6] M. J. Benito, D. J. Veale, O. FitzGerald, W. B. van den Berg, and B. Bresnihan, "Synovial tissue inflammation in early and late osteoarthritis," Annals of the Rheumatic Diseases, vol. 64, no. 9, pp. 1263-1267, 2005.

[7] H. Kato, A. Matsumine, T. Wakabayashi et al., "Large-scale gene expression profiles, differentially represented in osteoarthritic synovium of the knee joint using cDNA microarray technology," Biomarkers, vol. 12, no. 4, pp. 384-402, 2007.

[8] Y. Sun, D. R. Mauerhan, G. S. Firestein, B. J. Loeffler, E. N. Hanley, and H. E. Gruber, "Telomerase transduced osteoarthritis fibroblast-like synoviocytes display a distinct gene expression profile," The Journal of Rheumatology, vol. 36, no. 1, pp. 141-155, 2009.

[9] E. B. Hamilton, "Diseases associated with CPPD deposition disease," Arthritis \& Rheumatism, vol. 19, supplement 3, pp. 353357, 1976.

[10] G. J. Carroll, R. A. Stuart, J. A. Armstrong, P. D. Breidahl, and B. A. Laing, "Hydroxyapatite crystals are a frequent finding in osteoarthritic synovial fluid, but are not related to increased concentrations of keratan sulfate or interleukin $1 \beta$," The Journal of Rheumatology, vol. 18, no. 6, pp. 861-866, 1991.

[11] B. A. Derfus, J. B. Kurian, J. J. Butler et al., "The high prevalence of pathologic calcium crystals in pre-operative knees," The Journal of Rheumatology, vol. 29, no. 3, pp. 570-574, 2002.

[12] S. Nalbant, J. A. M. Martinez, T. Kitumnuaypong, G. Clayburne, M. Sieck, and H. R. Schumacher Jr., "Synovial fluid features and their relations to osteoarthritis severity: new findings from sequential studies," Osteoarthritis and Cartilage, vol. 11, no. 1, pp. 50-54, 2003. 
[13] M. Fuerst, L. Lammers, F. Schäfer et al., "Investigation of calcium crystals in OA knees," Rheumatology International, vol. 30, no. 5, pp. 623-631, 2010.

[14] Y. Sun, D. R. Mauerhan, P. R. Honeycutt et al., "Calcium deposition in osteoarthritic meniscus and meniscal cell culture," Arthritis Research and Therapy, vol. 12, no. 2, article R56, 2010.

[15] H. S. Cheung, "Biologic effects of calcium-containing crystals," Current Opinion in Rheumatology, vol. 17, pp. 336-340, 2005.

[16] Y. Sun, L. Wenger, C. E. Brinckerhoff, R. R. Misra, and H. S. Cheung, "Basic calcium phosphate crystals induce matrix metalloproteinase-1 through the Ras/mitogen-activated protein kinase/c-Fos/AP-1/metalloproteinase 1 pathway: involvement of transcription factor binding sites AP-1 and PEA-3," The Journal of Biological Chemistry, vol. 277, no. 2, pp. 1544-1552, 2002.

[17] M. P. Morgan, L. C. Whelan, J. D. Sallis, C. J. McCarthy, D. J. Fitzgerald, and G. M. McCarthy, "Basic calcium phosphate crystal-induced prostaglandin E2 production in human fibroblasts: role of cyclooxygenase 1 , cyclooxygenase 2 , and interleukin-1beta," Arthritis \& Rheumatism, vol. 50, no. 5, pp. 1642-1649, 2004.

[18] Y. Sun, X. R. Zeng, L. Wenger, and H. S. Cheung, "Basic calcium phosphate crystals stimulate the endocytotic activity of cells-inhibition by anti-calcification agents," Biochemical and Biophysical Research Communications, vol. 312, no. 4, pp. 10531059, 2003.

[19] M. Fuerst, J. Bertrand, L. Lammers et al., "Calcification of articular cartilage in human osteoarthritis," Arthritis \& Rheumatism, vol. 60, no. 9, pp. 2694-2703, 2009.

[20] T. Habata, H. Ohgushi, Y. Takakura et al., "Relationship between meniscal degeneration and element contents," Biological Trace Element Research, vol. 79, no. 3, pp. 247-256, 2001.

[21] K. P. H. Pritzker, "Counterpoint: hydroxyapatite crystal deposition is not intimately involved in the pathogenesis and progression of human osteoarthritis," Current Rheumatology Reports, vol. 11, no. 2, pp. 148-153, 2009.

[22] W. P. Tew, C. Mahle, J. Benavides, J. E. Howard, and A. L. Lehninger, "Synthesis and characterization of phosphocitric acid, a potent inhibitor of hydroxylapatite crystal growth," Biochemistry, vol. 19, no. 9, pp. 1983-1988, 1980.

[23] D. Nair, R. P. Misra, J. D. Sallis, and H. S. Cheung, "Phosphocitrate inhibits a basic calcium phosphate and calcium pyrophosphate dihydrate crystal-induced mitogen-activated protein kinase cascade signal transduction pathway," The Journal of Biological Chemistry, vol. 272, no. 30, pp. 18920-18925, 1997.

[24] H. S. Cheung, J. D. Sallis, and J. A. Struve, "Specific inhibition of basic calcium phosphate and calcium pyrophosphate crystalinduction of metalloproteinase synthesis by phosphocitrate," Biochimica et Biophysica Acta, vol. 1315, no. 2, pp. 105-111, 1996.

[25] Y. Sun, P. Reuben, L. Wenger, J. D. Sallis, K. D. Demadis, and H. S. Cheung, "Inhibition of calcium phosphate-DNA coprecipitates induced cell death by phosphocitrates," Frontiers in Bioscience, vol. 10, pp. 803-808, 2005.

[26] H. S. Cheung, J. D. Sallis, K. D. Demadis, and A. Wierzbicki, "Phosphocitrate blocks calcification-induced articular joint degeneration in a guinea pig model," Arthritis \& Rheumatism, vol. 54, no. 8, pp. 2452-2461, 2006.

[27] H. S. Cheung, "Calcium crystal effects on the cells of the joint: implications for pathogenesis of disease," Current Opinion in Rheumatology, vol. 12, no. 3, pp. 223-227, 2000.
[28] M. Ding, C. C. Danielsen, and I. Hvid, "The effects of bone remodeling inhibition by alendronate on three-dimensional microarchitecture of subchondral bone tissues in guinea pig primary osteoarthrosis," Calcified Tissue International, vol. 82, no. 1, pp. 77-86, 2008.

[29] L. C. Ward, R. Shankar, and J. D. Sallis, "A possible antiatherogenic role for phosphocitrate through modulation of low density lipoprotein uptake and degradation in aortic smooth muscle cells," Atherosclerosis, vol. 65, no. 1-2, pp. 117-124, 1987.

[30] H. E. Krug, "Fibroblasts from mice with progessive ankylosis proliferate excessively in response to transforming growth factor-beta 1," Journal of Investigative Medicine, vol. 46, no. 4, pp. 134-139, 1998.

[31] P. A. Turhanen, K. D. Demadis, S. Peräniemi, and J. J. Vepsäläinen, "A novel strategy for the preparation of naturally occurring phosphocitrate and its partially esterified derivatives," Journal of Organic Chemistry, vol. 72, no. 4, pp. 1468-1471, 2007.

[32] M. Pfaffl, H. H. D. Meyer, and H. Sauerwein, "Quantification of insulin-like growth factor-1 (IGF-1) mRNA: development and validation of an internally standardised competitive reverse transcription-polymerase chain reaction," Experimental and Clinical Endocrinology and Diabetes, vol. 106, no. 6, pp. 506-513, 1998.

[33] H. S. Cheung, "Phosphocitrate as a potential therapeutic strategy for crystal deposition disease," Current Rheumatology Reports, vol. 3, no. 1, pp. 24-28, 2001.

[34] M. M. Cooke, G. M. McCarthy, J. D. Sallis, and M. P. Morgan, "Phosphocitrate inhibits calcium hydroxyapatite induced mitogenesis and upregulation of matrix metalloproteinase-1, interleukin- $1 \beta$ and cyclooxygenase- 2 mRNA in human breast cancer cell lines," Breast Cancer Research and Treatment, vol. 79, no. 2, pp. 253-263, 2003.

[35] C. L. Hill, D. J. Hunter, J. Niu et al., "Synovitis detected on magnetic resonance imaging and its relation to pain and cartilage loss in knee osteoarthritis," Annals of the Rheumatic Diseases, vol. 66, no. 12, pp. 1599-1603, 2007.

[36] F. W. Roemer, Y. Zhang, J. Niu et al., “Tibiofemoral joint osteoarthritis: risk factors for MR-depicted fast cartilage loss over a 30-month period in the multicenter osteoarthritis study," Radiology, vol. 252, no. 3, pp. 772-780, 2009.

[37] D. A. Walsh, C. S. Bonnet, E. L. Turner, D. Wilson, M. Situ, and D. F. McWilliams, "Angiogenesis in the synovium and at the osteochondral junction in osteoarthritis," Osteoarthritis and Cartilage, vol. 15, no. 7, pp. 743-751, 2007.

[38] M. J. Del Rey, E. Izquierdo, S. Caja et al., "Human inflammatory synovial fibroblasts induce enhanced myeloid cell recruitment and angiogenesis through a hypoxia-inducible transcription factor $1 \alpha /$ vascular endothelial growth factor-mediated pathway in immunodeficient mice," Arthritis \& Rheumatism, vol. 60, no. 10, pp. 2926-2934, 2009.

[39] COX-2 Inhibitors, "New medication for arthritis pain," Mayo Clinic Health Letter, vol. 17, article 7, 1999.

[40] N. E. Lane, "Pain management in osteoarthritis: the role of COX-2 inhibitors," Journal of Rheumatology Supplement, vol. 24, no. 49, pp. 20-24, 1997.

[41] G. Saxler, F. Löer, M. von Knoch, F. von Knoch, and U. Hanesch, "Localization of the neurokinin1 receptor in hip joints of patients with painful osteoarthritis," Zeitschrift fur Orthopadie und Ihre Grenzgebiete, vol. 143, no. 4, pp. 424-430, 2005.

[42] K. Yamaguchi, T. Kugimiya, and T. Miyazaki, "Substance $\mathrm{P}$ receptor in U373 MG human astrocytoma cells activates 
mitogen-activated protein kinases ERK1/2 through Src," Brain Tumor Pathology, vol. 22, no. 1, pp. 1-8, 2005.

[43] T. M. O'Connor, J. O'Connell, D. I. O'Brien, T. Goode, C. P. Bredin, and F. Shanahan, "The role of substance P in inflammatory disease," Journal of Cellular Physiology, vol. 201, no. 2, pp. 167-180, 2004.

[44] R. E. Miller, P. B. Tran, R. Das et al., "CCR2 chemokine receptor signaling mediates pain in experimental osteoarthritis," Proceedings of the National Academy of Sciences of the United States of America, vol. 109, pp. 20602-20607, 2012.

[45] N. Busso, V. Péclat, A. So, and A. P. Sappino, "Plasminogen activation in synovial tissues: differences between normal, osteoarthritis, and rheumatoid arthritis joints," Annals of the Rheumatic Diseases, vol. 56, no. 9, pp. 550-557, 1997.

[46] O. Slot, N. Brünner, H. Locht, P. Oxholm, and R. W. Stephens, "Soluble urokinase plasminogen activator receptor in plasma of patients with inflammatory rheumatic disorders: increased concentrations in rheumatoid arthritis," Annals of the Rheumatic Diseases, vol. 58, no. 8, pp. 488-492, 1999.

[47] W. Schwab, J. M. Gavlik, T. Beichler et al., "Expression of the urokinase-type plasminogen activator receptor in human articular chondrocytes: association with caveolin and $\beta 1$-integrin," Histochemistry and Cell Biology, vol. 115, no. 4, pp. 317-323, 2001.

[48] A. Trabandt, W. K. Aicher, R. E. Gay, V. P. Sukhatme, H. G. Fassbender, and S. Gay, "Spontaneous expression of immediatelyearly response genes c-fos and egr-1 in collagenase-producing rheumatoid synovial fibrolasts," Rheumatology International, vol. 12, no. 2, pp. 53-59, 1992.

[49] Y. Sun, L. Wenger, C. E. Brinckerhoff, R. R. Misra, and H. S. Cheung, "Basic calcium phosphate crystals induce matrix metalloproteinase-1 through the Ras/mitogen-activated protein kinase/c-Fos/AP-1/metalloproteinase 1 pathway: involvement of transcription factor binding sites AP-1 and PEA-3," The Journal of Biological Chemistry, vol. 277, no. 2, pp. 1544-1552, 2002.

[50] H. Kawasaki, K. Komai, M. Nakamura et al., "Human weel kinase is directly transactivated by and increased in association with c-Fos/AP-1: rheumatoid synovial cells overexpressing these genes go into aberrant mitosis," Oncogene, vol. 22, no. 44, pp. 6839-6844, 2003.

[51] T. Okabe, Y. Ohmori, A. Tanigami et al., "Detection of gene expression in synovium of patients with osteoarthritis using a random sequencing method," Acta Orthopaedica, vol. 78, no. 5, pp. 687-692, 2007.

[52] W. H. Busby Jr., S. A. Yocum, M. Rowland et al., "Complement $1 \mathrm{~s}$ is the serine protease that cleaves IGFBP-5 in human osteoarthritic joint fluid," Osteoarthritis and Cartilage, vol. 17, no. 4, pp. 547-555, 2009.

[53] D. R. Clemmons, W. H. Busby Jr., A. Garmong et al., "Inhibition of insulin-like growth factor binding protein 5 proteolysis in articular cartilage and joint fluid results in enhanced concentrations of insulin-like growth factor 1 and is associated with improved osteoarthritis," Arthritis and Rheumatism, vol. 46, no. 3, pp. 694-703, 2002. 


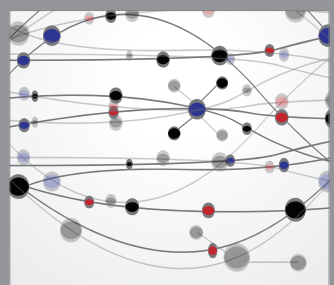

The Scientific World Journal
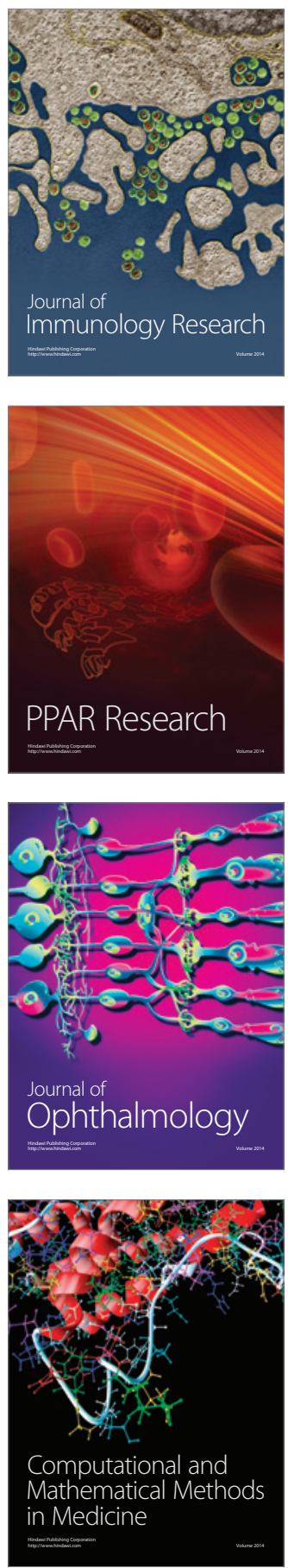

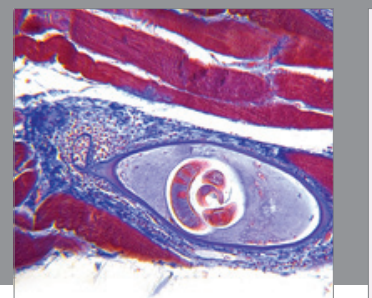

Gastroenterology

Research and Practice
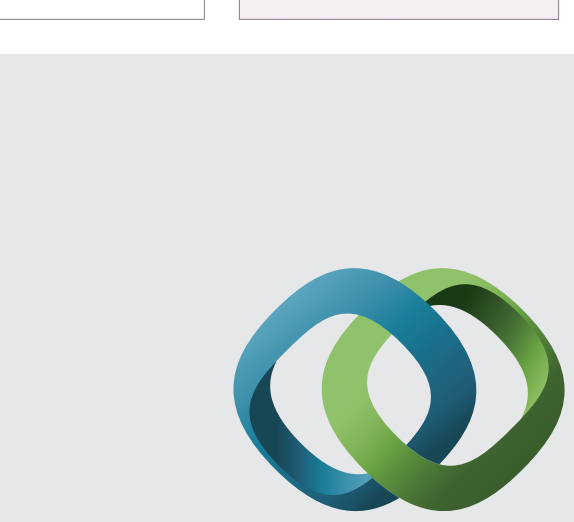

\section{Hindawi}

Submit your manuscripts at

http://www.hindawi.com
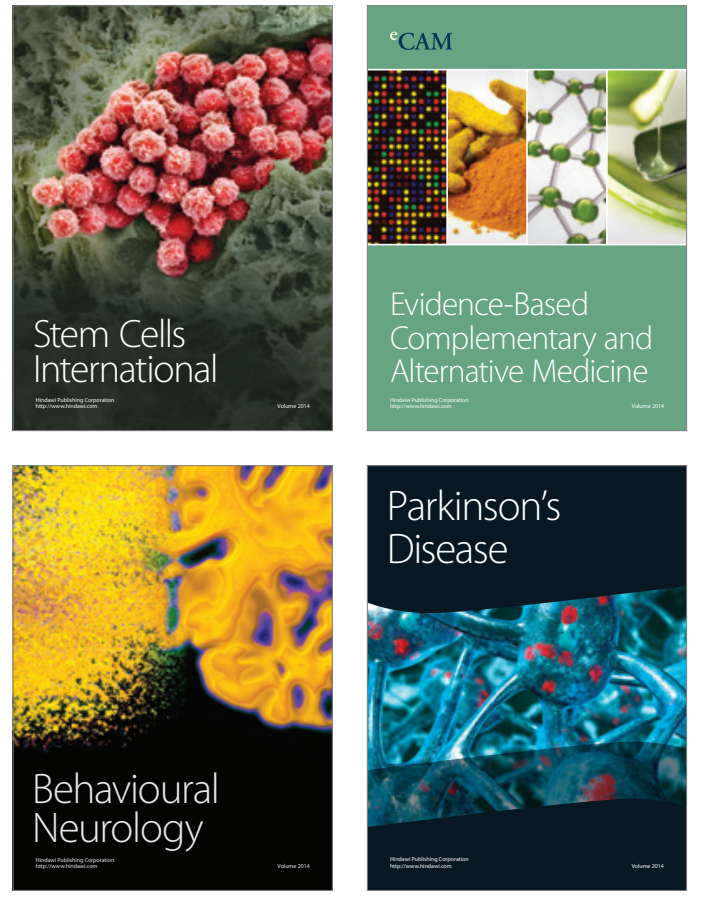
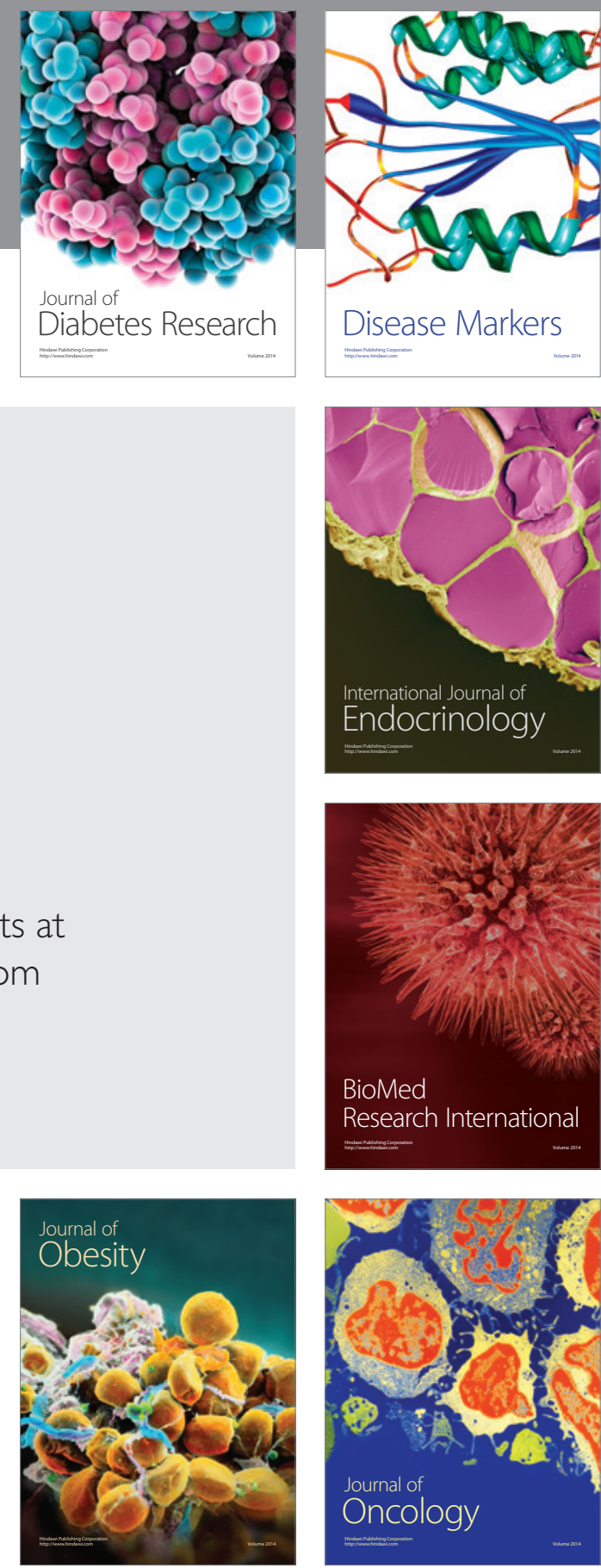

Disease Markers
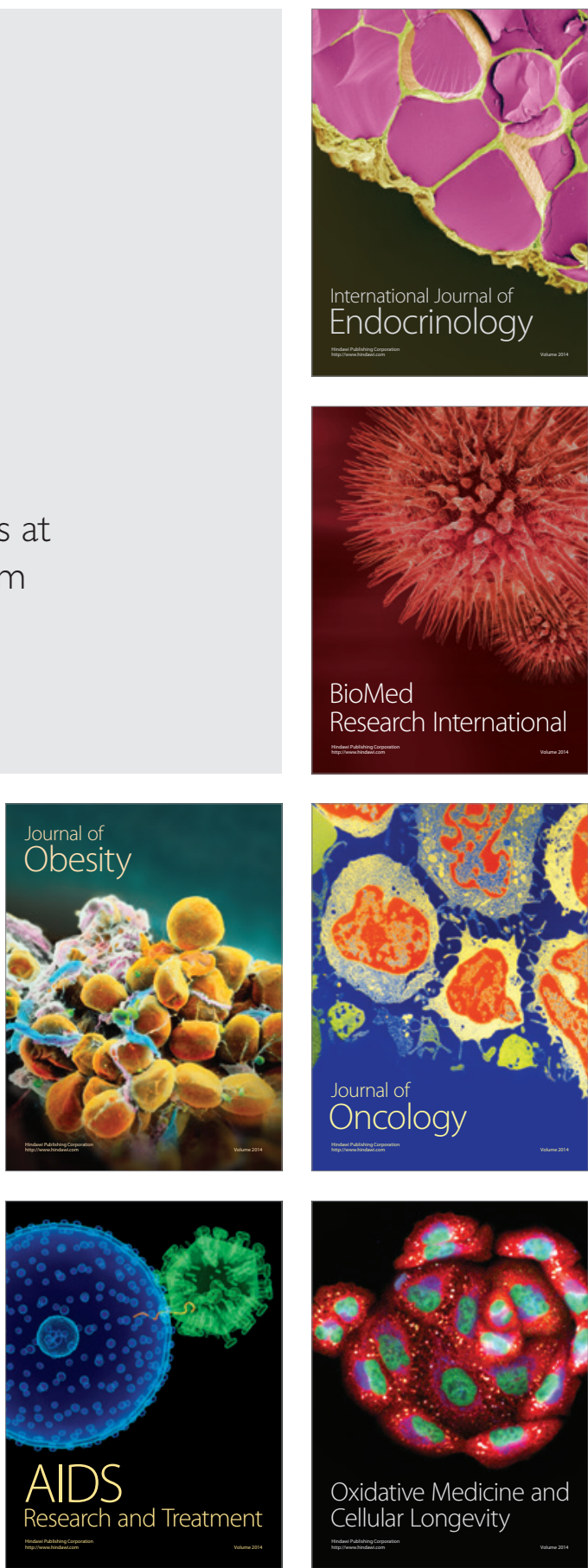